\title{
A Toolchain for the Analysis of Hydrogen in Materials at the Atomic Scale
}

\author{
Peter Johann Felfer ${ }^{*}$
}

1. Department of Materials Science, Institute for General Materials Properties, Friedrich-Alexander Universität Erlangen-Nürnberg, Germany

* Corresponding author: peter.felfer@fau.de

The analysis of amount and location of $\mathrm{H}$ in solids is highly challenging due to a number of reasons [1]: firstly, $\mathrm{H}$ is the lightest of elements and therefore has a nucleus with limited scattering power, making imaging with e.g. electrons. Secondly, $\mathrm{H}$ is contained in many materials microscopes are made of, often creating spurious signals. Furthermore, $\mathrm{H}$ is highly mobile in many solids and therefore may move during the imaging process even at moderate temperatures. For example, in common metals $\mathrm{H}$ may move significantly already at and below room temperature.

Atom probe tomography (APT) presents a method where paths to overcome these challenges exist. The low scattering power of the $\mathrm{H}$ nucleus is not an issue for APT, since the principle of time-of-flight mass spectrometry is, with some minor limitations, equally applicable to all chemical elements. The problem of mobility during the measurement can e.g. be addressed by choosing low enough temperatures during the experiment and high measurement rates. The problem of spurious $\mathrm{H}$ on the other hand has so far not generally been addressed. In all almost data from currently operating atom probe instruments, $\mathrm{H}$ can be found in varying amounts. This spurious $\mathrm{H}$ is attributed to $\mathrm{H}$ diffusing in from the chamber walls made from stainless steel.

For the problem of spurious $\mathrm{H}$ occluding the $\mathrm{H}$ to be analyzed, a workaround exists. Since the mass spectrometry principle allows for the distinction between ${ }^{1} \mathrm{H}$ and ${ }^{2} \mathrm{H}$ (deuterium, D), experiments where $\mathrm{H}$ is artificially introduced can be carried out using D with its rather low natural abundance of 115 ppm rather than ${ }^{1} \mathrm{H}$. Through this method, a variety of studies on $\mathrm{H}$ in materials were carried out. In these studies, the problem of $\mathrm{D}$ mobility before the analysis was either not present because stable hydrides were investigated [2], or fast transfers from in-situ charging stations was used [3] or the samples were cryo-cooled after $\mathrm{H}$ charging [4].

Here, we present the implementation of a versatile, fully cryo-cooled analysis toolchain to preserve the original distribution of $\mathrm{H}$ in materials as true to the initial state as possible. In the authors lab, the analysis toolchain includes a plunge freezing unit with cryogenic electropolishing of metallic samples using fully deuterated chemicals (fig. 1a), or alternatively cryogenic focused ion beam preparation (fig. 1b). The sample preparation can be extended by using a cryo-cooled electron beam coating unit with broad ion beam etching and indirect ion beam deposition capability. The samples can be transferred between the various tools and the atom probe instruments and the field ion microscope in the lab with a cryo-shuttle (fig. 1c), which is actively cooled by through liquid nitrogen. Currently, this setup is operational with a modified version of a commercial CAMECA LEAP 4000X HR atom probe (fig. 1d). Experiments have been carried out on D at grain boundaries and phase interfaces in Fe based materials. Strong signals of D at these interfaces have been detected, for the first time allowing the quantification of $\mathrm{H}$ through the $\mathrm{D}$ route at grain boundaries, which are the most relevant microstructural features regarding $\mathrm{H}$ induced fracture of metallic materials. Furthermore, this toolchain can be used to suppress unwanted artefacts from focused ion beam preparation. An important example is the liquid metal embrittlement that is caused by Ga penetrating into Al grain boundaries. This does not occur at cryogenic temperatures. 
To analyze materials where the $\mathrm{H}$ has been incorporated through processes that cannot be mimicked in the lab however, an analysis toolchain that can preserve the original distribution of $\mathrm{H}$ in the material as well as not be influenced by residual $\mathrm{H}$ is highly desirable. Such analysis tasks include the determination of amount and location of residual $\mathrm{H}$ in metallic materials after metallurgical processes or the analysis of $\mathrm{H}$ in interstellar matter. To achieve this, the author is currently building an atom probe instrument that incorporates the missing two links: an actively cooled sample storage system and a main analysis chamber with ultra-low levels of residual H (fig. 2a). This instrument can be interfaced with the above cryo sample preparation instrumentation similar to the existing commercial instrument.

To allow for sample storage at cryogenic temperatures, a single-stage closed cycle Gifford-McMahon cryocooler with $12 \mathrm{~W}$ cooling power is interfaced to the sample carousel. This allows for cooling to temperatures around $50 \mathrm{~K}$. At this temperature, volume diffusion even of $\mathrm{H}$ is effectively suppressed. This is combined with a main analysis chamber manufactured from Ti [5] (fig. 2b), equipped with a 1.5W@4K cryocooler, where ultra-low $\mathrm{H}$ levels are to be expected. However, the ultimate vacuum performance of the instrument and with it the $\mathrm{H}$ analysis capability have not yet been tested but first results will be presented at the Portland meeting [6].

\section{References:}

[1] R. Gemma et al., Journal of Alloys and Compounds 509 (2011), p. S872.

DOI: 10.1016/J.JALLCOM.2010.11.122

[3] J. Takahashi et al., Scripta Materialia 63/3 (2010), p. 261, DOI :10.1016/j.scriptamat.2010.03.012

[4] Y. Chen et al., Science 355 (2017), p. 1196, DOI: 10.1126/science.aal2418

[5] M. Takeda et al., Applied Surface Science 258/4 (2011), p. 1405, DOI: 10.1016/j.apsusc.2011.09.092

[6] This work is supported by the European Research Council through the Starting Grant HydMet. The author also acknowledges the use of facilities of the Center for Nanoanalysis and Electron Microscopy at the Friedrich-Alexander Universität Erlangen-Nürnberg.

a)

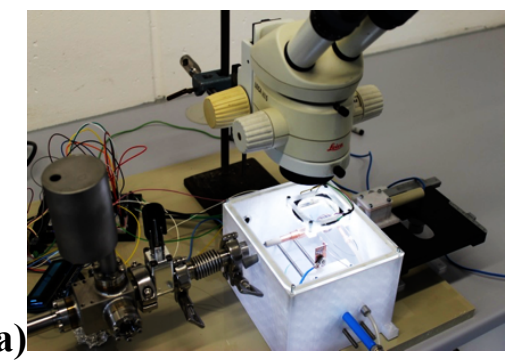

b)

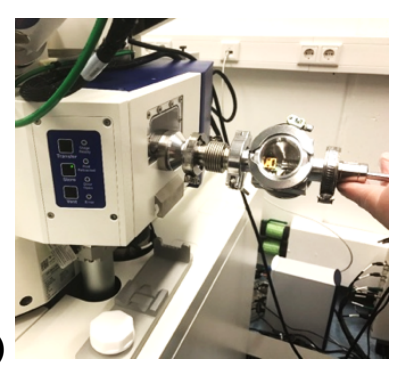

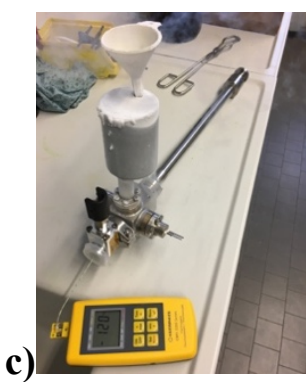

d)

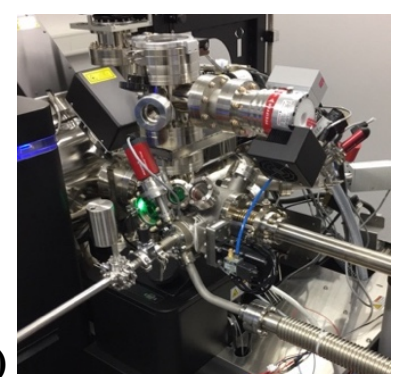

Figure 1. a) Cryo electropolishing unit. b) Cryo-FIB with transfer unit. c) Commercial LEAP 4000X HR with cryo transfer unit. d) Cryo shuttle.

a)

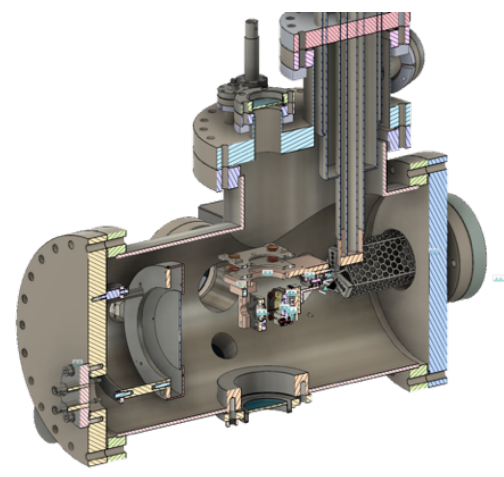

b)

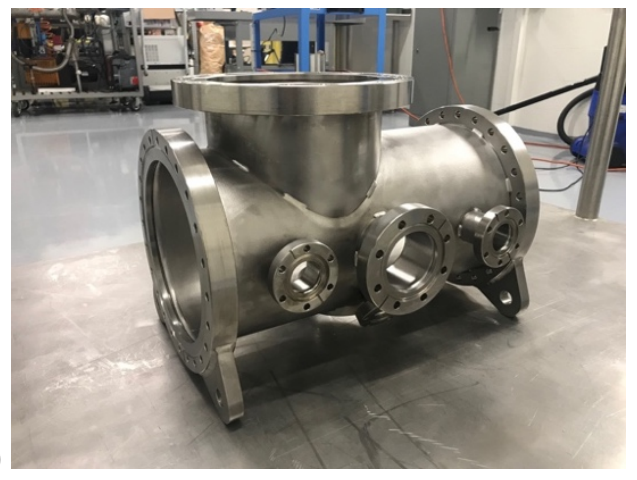

Figure 2. a) Design of an ultra-low $\mathrm{H}$ background atom probe instrument. b) Photograph of the Ti main chamber. 